\title{
Change in Internal Energy and Enthalpy of Spinning Black Holes in XRBs and AGN Using Spin Parameter $a^{*}=0.9$
}

\author{
Dipo Mahto', Alok Ranjan², Niharika Kumari ${ }^{2}$ \\ ${ }^{1}$ Department of Physics, Marwari College, T. M. B. University, Bhagalpur, India \\ ${ }^{2}$ Department of Physics, T. M. B. University, Bhagalpur, India \\ Email:dipomahto@hotmail.com
}

How to cite this paper: Mahto, D., Ranjan, A. and Kumari, N. (2017) Change in Internal Energy and Enthalpy of Spinning Black Holes in XRBs and AGN Using Spin Parameter $a^{*}=0.9$ International Journal of Astronomy and Astrophysics, 7, 38-44. https://doi.org/10.4236/ijaa.2017.71004

Received: February 5, 2017

Accepted: March 28, 2017

Published: March 31, 2017

Copyright $\odot 2017$ by authors and Scientific Research Publishing Inc. This work is licensed under the Creative Commons Attribution International License (CC BY 4.0). http://creativecommons.org/licenses/by/4.0/

\begin{abstract}
In the present research work, we have proposed a model for the change in the internal energy and enthalpy of the spinning black holes using first law of black holes in the case of spin parameter $a^{*}=0.9$ and calculated their values in XRBs and AGN.
\end{abstract}

\section{Keywords}

Surface Gravity, Enthalpy, Spinning Black Holes and AGN

\section{Introduction}

Classically, the black hole created after the death of red giant star is the perfect absorber like a black body and does not emit anything; their temperature is absolute zero. However, in quantum theory, black holes emit Hawking radiation with a perfect thermal spectrum [1] [2] [3]. According to the GR, the black hole is a solution of Einstein's gravitational field equations in the absence of matter that describes the space time around a gravitationally collapsed star [4]. Mahto et al. proposed a model for the change in the internal energy and enthalpy of the black holes using first law of black holes which showed that the change in internal energy and enthalpy was the manifestations of same thing at constant pressure and volume [5]. This work is extended in the case of spin parameter $a^{*}=1 / 2$ with the calculations for the change in the internal energy and enthalpy of the different test of spinning black holes in XRBs [6].

In the present work, we have proposed a model for the change in the internal energy and enthalpy of the spinning black holes using first law of black holes for the case of spin parameter $a^{*}=0.9$ and calculated their values in XRBs and AGN. 


\section{Method}

The change in internal energy and enthalpy of black holes with corresponding change in the radius of the event horizon of black holes is given by [6]

$$
\mathrm{d} H=\mathrm{d} U=\kappa R_{b h} \mathrm{~d} R_{b h} .
$$

The change in internal energy and enthalpy of spinning black holes will have different values in compared with that of non-spinning black holes, because the surface gravity of a black hole is given by the Kerr solution [7].

$$
\kappa=\frac{\left(M^{4}-J_{H}^{2}\right)^{1 / 2}}{2 M\left\{M^{2}+\left(M^{4}-J_{H}^{2}\right)^{1 / 2}\right\}}
$$

where

$$
J_{H}=a_{*} M^{2} .
$$

The surface gravity $(\kappa)$ can be thought of roughly as the acceleration at horizon of black hole and it has the same role in the black hole mechanics as the temperature in the ordinary laws of thermodynamics [4]. According to the zeroth law of classical black hole mechanics, the surface gravity $(\kappa)$ of a black hole is constant on horizon and the surface gravity tends to zero when the magnitude of charge of a black holes becomes equal to the mass of black holes [8].

Wang and Ding-Xiong have shown that the angular velocity $\left(\Omega_{H}\right)$ evolves in a non-monotonous way in the case of thin disk-pure-accretion attaining a maximum at $a^{*}=0.994$ and turns out to depend on the radial gradient of $\Omega_{p}$ near the BH horizon [9]. One black hole at the heart of galaxy NGC1365 is turning at $84 \%$ of the speed of light. It has reached the cosmic speed limit and cannot spin any faster without revealing its singularity [10].

For convenience, let us assume

$$
a^{*}=0.9 \text {. }
$$

Putting (3) and (4) in Equation (2) and solving, we have

$$
\kappa=\frac{.1517}{M} \text {. }
$$

Putting the above value in Equation (1)

$$
\begin{gathered}
\mathrm{d} H=\mathrm{d} U=\frac{0.1517}{M} R_{b h} \mathrm{~d} R_{b h} \\
\mathrm{~d} H / \mathrm{d} R_{b h}=\mathrm{d} U / \mathrm{d} R_{b h}=\frac{0.1517}{M} R_{b h} .
\end{gathered}
$$

The $e q^{n}$ (7) gives the change in internal energy as well as enthalpy with respect to corresponding change in the radius of the event horizon in terms of the mass and event horizon of spinning black holes.

\section{Data in Support of Mass of the Sun and Black Holes}

Mass of sun $\left(M_{\odot}\right)=1.99 \times 10^{30} \mathrm{~kg}$, [11]. There are two categories of black holes classified on the basis of their masses clearly very distinct from each other, with 
very different masses $M \sim 5-20 M_{\odot}$ for stellar - mass black holes in X-ray binaries and $M \sim 10^{6}-10^{9.5} M_{\odot}$ for super massive black holes in Galactic nuclei [11]. The other data in the support of black holes can be seen in the references [12] [13] [14] [15].

\section{Results and Discussion}

In the present work, we have derived an expression for the change in the internal energy and enthalpy of the spinning black holes taking an account the first law of black hole mechanics for the case of spin parameter $a^{*}=0.9$ and calculated their values in XRBs and AGN and plotted the graphs as per Figure $1 \&$ Figure 2.

For the angular spin $\left(a^{*}=0.9\right)$, we have finally obtained the change in internal energy and enthalpy of spinning black holes for different values of mass and event horizon as:

$$
\mathrm{d} H / \mathrm{d} R_{b h}=\mathrm{d} U / \mathrm{d} R_{b h}=\frac{0.1517}{M} R_{b h} .
$$

From the graph plotted between the change in internal energy and enthalpy w.r.t. the change in the event horizon and different values of mass and event horizon in XRBs and AGN, it is obvious that the change in internal energy and enthalpy w.r.t. the change in the event horizon remains the same. The change in the internal energy and enthalpy calculated for each black hole candidates in both categories of spinning black holes XRBs and AGN are exactly the same which are clear from data in Table $1 \&$ Table 2. From the graph plotted between the mass of different black holes and corresponding change in the internal energy/enthalpy, we observe that there is no variation in the change in enthalpy and

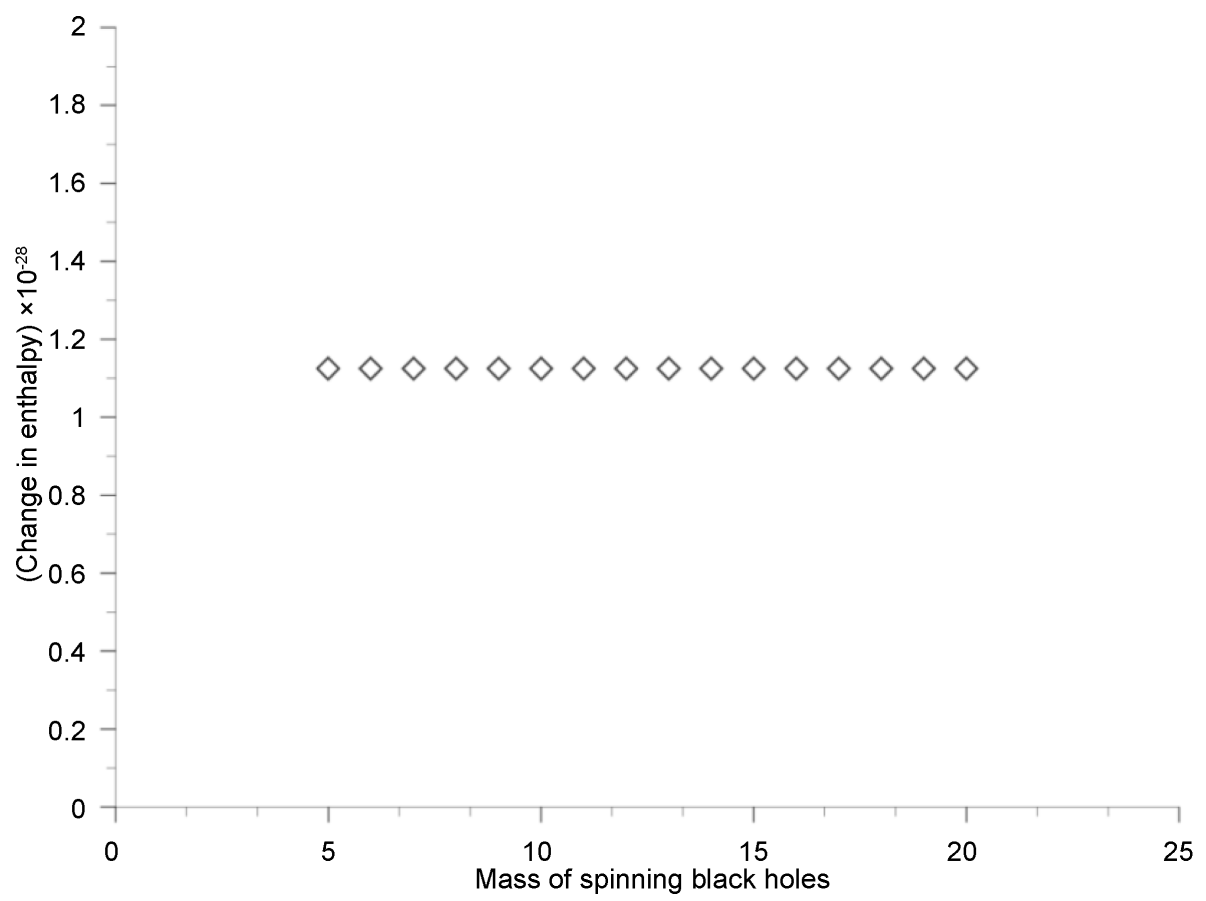

Figure 1. The graph plotted between the change in enthalpy/internal energy of spinning black holes with corresponding change in the radius of event horizon in XRBs. 


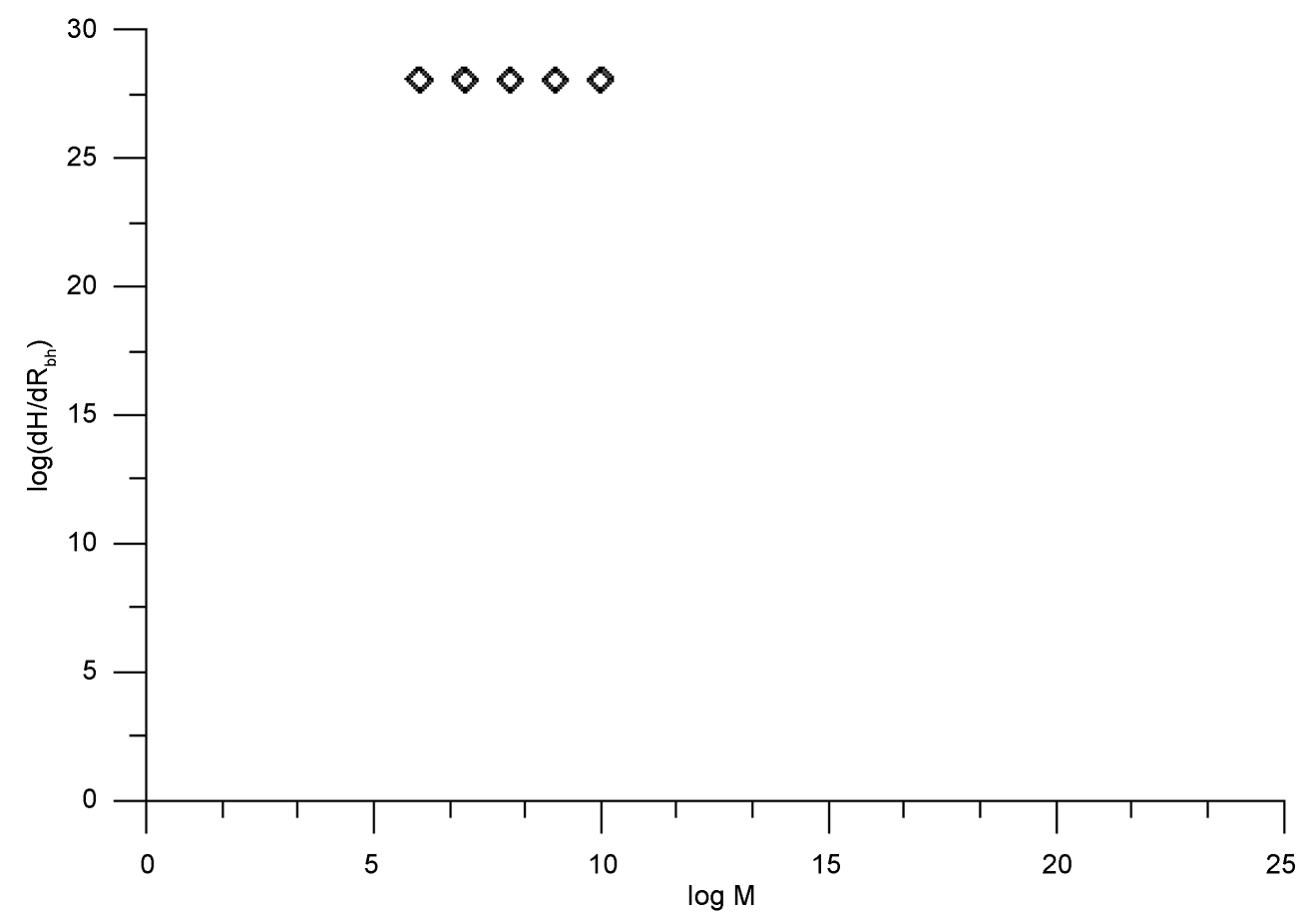

Figure 2. The graph plotted between the change in enthalpy/internal energy of spinning black holes with corresponding change in the radius of event horizon in AGN.

Table 1. Change in enthalpy and internal energy of spinning black holes in XRBs using spin parameter $a^{*}=0.9$.

\begin{tabular}{cccc}
\hline Sl. No. $(M)$ in terms of the solar mass $\left(M_{\odot}\right)$ & $\begin{array}{c}R_{b h}=1475\left(M / M_{\odot}\right) \\
\text { (in meter) }\end{array}$ & $\mathrm{d} H / \mathrm{d} R_{b h}=\mathrm{d} U / \mathrm{d} R_{b h}=\frac{0.1517}{M} R_{b h}$ \\
\hline 1 & 5 & 7375 & $1.1244 \times 10^{-28}$ \\
2 & 6 & 8850 & $1.1244 \times 10^{-28}$ \\
3 & 7 & 10,325 & $1.1244 \times 10^{-28}$ \\
4 & 8 & 11,800 & $1.1244 \times 10^{-28}$ \\
5 & 9 & 13,275 & $1.1244 \times 10^{-28}$ \\
6 & 10 & 14,750 & $1.1244 \times 10^{-28}$ \\
7 & 11 & 16,225 & $1.1244 \times 10^{-28}$ \\
8 & 12 & 17,700 & $1.1244 \times 10^{-28}$ \\
9 & 13 & 19,175 & $1.1244 \times 10^{-28}$ \\
10 & 14 & 20,650 & $1.1244 \times 10^{-28}$ \\
11 & 15 & 22,125 & $1.1244 \times 10^{-28}$ \\
12 & 16 & 23,600 & $1.1244 \times 10^{-28}$ \\
13 & 17 & 25,075 & $1.1244 \times 10^{-28}$ \\
14 & 18 & 26,550 & $1.1244 \times 10^{-28}$ \\
15 & 19 & 28,025 & $1.1244 \times 10^{-28}$ \\
16 & 20 & 29,500 & $1.1244 \times 10^{-28}$ \\
\hline
\end{tabular}

internal energy with change in mass/event horizon of the black holes.

Equation (7) shows that the change in enthalpy and internal energy with change in mass/event horizon of the black holes is directly proportional to the 
Table 2. Change in enthalpy and internal energy of spinning black holes in AGN using spin parameter $a^{*}=0.9$.

\begin{tabular}{|c|c|c|c|}
\hline Sl. No. & $\begin{array}{c}\text { Mass of BHs }(M) \\
\text { in terms of the solar mass }\left(M_{\odot}\right)\end{array}$ & $\begin{array}{c}R_{b h}=1475 \times\left(M / M_{\odot}\right) \\
(\text { in meter })\end{array}$ & $\mathrm{d} H / \mathrm{d} R_{b h}=\mathrm{d} U / \mathrm{d} R_{b h}=\frac{0.1517}{M} R_{b h}$ \\
\hline 1 & $1 \times 10^{6}$ & $1.475 \times 10^{9}$ & $1.1244 \times 10^{-28}$ \\
\hline 2 & $2 \times 10^{6}$ & $2.950 \times 10^{9}$ & $1.1244 \times 10^{-28}$ \\
\hline 3 & $3 \times 10^{6}$ & $4.425 \times 10^{9}$ & $1.1244 \times 10^{-28}$ \\
\hline 4 & $4 \times 10^{6}$ & $5.900 \times 10^{9}$ & $1.1244 \times 10^{-28}$ \\
\hline 5 & $5 \times 10^{6}$ & $7.375 \times 10^{9}$ & $1.1244 \times 10^{-28}$ \\
\hline 6 & $6 \times 10^{6}$ & $8.850 \times 10^{9}$ & $1.1244 \times 10^{-28}$ \\
\hline 7 & $7 \times 10^{6}$ & $10.320 \times 10^{9}$ & $1.1244 \times 10^{-28}$ \\
\hline 8 & $8 \times 10^{6}$ & $11.800 \times 10^{9}$ & $1.1244 \times 10^{-28}$ \\
\hline 9 & $9 \times 10^{6}$ & $13.270 \times 10^{9}$ & $1.1244 \times 10^{-28}$ \\
\hline 10 & $1 \times 10^{7}$ & $1.475 \times 10^{10}$ & $1.1244 \times 10^{-28}$ \\
\hline 11 & $2 \times 10^{7}$ & $2.950 \times 10^{10}$ & $1.1244 \times 10^{-28}$ \\
\hline 12 & $3 \times 10^{7}$ & $4.425 \times 10^{10}$ & $1.1244 \times 10^{-28}$ \\
\hline 13 & $4 \times 10^{7}$ & $5.900 \times 10^{10}$ & $1.1244 \times 10^{-28}$ \\
\hline 14 & $5 \times 10^{7}$ & $7.375 \times 10^{10}$ & $1.1244 \times 10^{-28}$ \\
\hline 15 & $6 \times 10^{7}$ & $8.850 \times 10^{10}$ & $1.1244 \times 10^{-28}$ \\
\hline 16 & $7 \times 10^{7}$ & $10.320 \times 10^{10}$ & $1.1244 \times 10^{-28}$ \\
\hline 17 & $8 \times 10^{7}$ & $11.800 \times 10^{10}$ & $1.1244 \times 10^{-28}$ \\
\hline 18 & $9 \times 10^{7}$ & $13.270 \times 10^{10}$ & $1.1244 \times 10^{-28}$ \\
\hline 19 & $1 \times 10^{8}$ & $1.475 \times 10^{11}$ & $1.1244 \times 10^{-28}$ \\
\hline 20 & $2 \times 10^{8}$ & $2.950 \times 10^{11}$ & $1.1244 \times 10^{-28}$ \\
\hline 21 & $3 \times 10^{8}$ & $4.425 \times 10^{11}$ & $1.1244 \times 10^{-28}$ \\
\hline 22 & $4 \times 10^{8}$ & $5.900 \times 10^{11}$ & $1.1244 \times 10^{-28}$ \\
\hline 23 & $5 \times 10^{8}$ & $7.375 \times 10^{11}$ & $1.1244 \times 10^{-28}$ \\
\hline 24 & $6 \times 10^{8}$ & $8.850 \times 10^{11}$ & $1.1244 \times 10^{-28}$ \\
\hline 25 & $7 \times 10^{8}$ & $10.320 \times 10^{11}$ & $1.1244 \times 10^{-28}$ \\
\hline 26 & $8 \times 10^{8}$ & $11.800 \times 10^{11}$ & $1.1244 \times 10^{-28}$ \\
\hline 27 & $9 \times 10^{8}$ & $13.270 \times 10^{11}$ & $1.1244 \times 10^{-28}$ \\
\hline 28 & $1 \times 10^{9}$ & $1.475 \times 10^{12}$ & $1.1244 \times 10^{-28}$ \\
\hline 29 & $2 \times 10^{9}$ & $2.950 \times 10^{12}$ & $1.1244 \times 10^{-28}$ \\
\hline 30 & $3 \times 10^{9}$ & $4.425 \times 10^{12}$ & $1.1244 \times 10^{-28}$ \\
\hline
\end{tabular}

radius of the event horizon and inversely proportional to mass of the black holes. Hence these two factors mass $(M)$ and the event horizon $\left(R_{b h}\right)$ adjust themselves in such a way that they give the constant values for the change in energy $(\delta H)$ of the black holes in each case. Figure $1 \&$ Figure 2 show the graph plotted between the mass of different $\mathrm{BHs}$ and corresponding the change in enthalpy and internal energy with change in mass/event horizon of the black holes in AGN of the black holes. In addition to this explanation, while observing the ratio of the radius of the event horizon and mass of the black hole for each case, gives con- 
stant values existing either in XRBs or AGN. The fourth column of the table shows that the change in internal energy/enthalpy for all the spinning black holes remain constant i.e. $\delta H=\delta U=$ constant. This means that it follows the principle of conservation of enthalpy and internal energy of spinning black holes just like the principle of conservation of energy.

\section{Conclusions}

1) For the angular spin $\left(a^{*}=0.9\right)$, the change in internal energy and enthalpy of spinning black holes in terms of mass and event horizon as:

$$
\mathrm{d} H / \mathrm{d} R_{b h}=\mathrm{d} U / \mathrm{d} R_{b h}=\frac{0.1517}{M} R_{b h} .
$$

2) The change in enthalpy and internal energy calculated with the help of above equation as given in the conclusion (1) for each black hole candidate in both categories of XRBs and AGN are exactly the same, showing the constant change in enthalpy and internal energy equal to $1.1244 \times 10^{-28}$ joule.

3) This agrees with the principle of conservation of the enthalpy and internal energy just like the principle of conservation of the energy.

4) The enthalpy and internal energy have the same role as the energy in the case of spinning black holes.

5) The enthalpy and internal energy of spinning black holes are the manifestation of the same thing.

\section{References}

[1] Hawking, S.W. (1975) Particle Creation by Black Holes. Communications in Mathematical Physic, 43, 199-220. https://doi.org/10.1007/BF02345020

[2] Hawking, S.W. (1974) Black Hole Explosions? Nature, 248, 30-31. https://doi.org/10.1038/248030a0

[3] Wald, R.M. (2001) The Thermodynamics of Black Holes. Living Reviews in Relativity, 4, 6. https://doi.org/10.12942/lrr-2001-6

[4] Dabholkar, A. (2005) Black Hole Entropy in String Theory-A Window into the Quantum Structure of Gravity. Current Science, 89, 25.

[5] Mahto, D., Singh, A.K., Ram, M. and Vineeta, K. (2013) Change in Internal Energy \& Enthalpy of the Black Holes. International Journal of Astrophysics and Space Science, 1, 20-22. https://doi.org/10.11648/j.ijass.20130104.12

[6] Mahto, D., Singh, A.K. and Kumari, N. (2016) Enthalpy of the Spinning Black Holes with Half Spin Parameter in XRBs. International Journal of Astronomy and Astrophysics, 6, 328-333. http://www.scirp.org/journal/ijaa https://doi.org/10.4236/ijaa.2016.63027

[7] Bardeen, J.M., Carter, B. and Hawking, S.W. (1973) The Four Laws of Black Hole Mechanics. Communications in Mathematical Physics, 31, 161-170. https://doi.org/10.1007/BF01645742

[8] Transchen, J. (2000) An Introduction to Black Hole Evaporation. arXiv:gr-qc/0010055V1

[9] Wang D.-X. (2000) Relations between Black Hole Spin and Angular Velocity of Accreting Particles near the Horizon. General Relativity and Gravitation, 32, 553-564. https://doi.org/10.1023/A:1001908131543 
[10] Cain, R. (2015) How Fast Do Black Holes Spin? Universe Today.

[11] Narayan, R. (2005) Black Holes in Astrophysics. New Journal Physics, 7, 1-31. arXiv:gr-qc/0506078v1 https://doi.org/10.1088/1367-2630/7/1/199

[12] Ferrarese, L. and Ford, H. (2005) Super Massive Black Holes in Galactic Nuclei: Past Present \& Future Research. Space Science Reviews, 116, 523-624. https://doi.org/10.1007/s11214-005-3947-6

[13] Madejski, G. (2003) Black Holes in Active Galactic Nuclei. SLAC-PUB-9702. https://doi.org/10.2172/812998

[14] Sargent, W.L.W. (1978) Dynamical Evidence for a Central Mass Concentration in the Galaxy M87. The Astrophysical Journal, 221, 731-744. https://doi.org/10.1086/156077

[15] Blandford, R.D. (1999) Recent Results on Active Galactic Nuclei. Astrophysics and Space Science, 261, 245-252. https://doi.org/10.1023/A:1002093710287

Submit or recommend next manuscript to SCIRP and we will provide best service for you:

Accepting pre-submission inquiries through Email, Facebook, LinkedIn, Twitter, etc. A wide selection of journals (inclusive of 9 subjects, more than 200 journals) Providing 24-hour high-quality service User-friendly online submission system Fair and swift peer-review system Efficient typesetting and proofreading procedure Display of the result of downloads and visits, as well as the number of cited articles Maximum dissemination of your research work

Submit your manuscript at: http://papersubmission.scirp.org/ Or contact ijaa@scirp.org 